\title{
Pseudomyogenic hemangioendothelioma: a little-known tumor
}

\author{
Rui Pedro Santos ${ }^{1 凶}$, Sofia Carvalho², Joana Gomes ${ }^{1}$, Joana Perdal
}

\begin{abstract}
Pseudomyogenic hemangioendothelioma (PHE) is a rare indolent vascular tumor that typically has a multifocal presentation and involves multiple tissue planes. This report describes a 34-year-old man with multiple infiltrated brown papules and plaques on his left leg that had evolved for 6 months. The skin biopsy revealed a dermal and subcutaneous neoplasm composed of fascicles of spindle cells with atypia and epithelioid cells with prominent nucleoli and abundant eosinophilic cytoplasm. There was no evidence of necrosis, and the mitotic rate was low. There was strong reactivity with cytokeratin $A E_{1} / A_{3}$, ERG, and FLl1, multifocal reactivity with smooth muscle actin, and focal reactivity with $C_{3}$. There was no expression of keratin MNF116, CAM5.2, CD 34, CAMTA1, $\mathrm{S}_{100}$ protein, epithelial membrane antigen, melan-A, HMB-45, factor XIIla, HHV8, or CD10. The nuclei of neoplastic cells showed intact expression of INl1. The clinical, histological, and immunophenotypical aspects were consistent with a diagnosis of PHE. A lower limb CT scan showed lesions in the skin, muscle, and bone planes. The patient was sent to an oncology center, where he maintains regular clinical and imagiological follow-up.
\end{abstract}

Keywords: pseudomyogenic hemangioendothelioma, epithelioid sarcoma-like, vascular neoplasm, skin, soft tissue, bone

Received: 4 March 2018 | Returned for modification: 20 July 2018 | Accepted: 27 August 2018

\section{Introduction}

Pseudomyogenic hemangioendothelioma (PHE)-previously referred to as a fibroma-like variant of epithelioid sarcoma, given its morphological similarity to epithelioid sarcoma -is a rare and recently described endothelial tumor. According to the World Health Organization (WHO), PHE is a tumor of intermediate malignancy that rarely metastasizes (1). PHE is typically found in young adults, especially males $(2,3)$. Although it can be found in several locations, this tumor typically affects the extremities (mainly the lower limbs). It tends to be multifocal and to involve multiple tissue planes in the same anatomical region.

The case presented here of a young man diagnosed with PHE illustrates the importance of an adequate differential diagnosis in order to preserve the quality of life of patients whose PHE can be mistaken for more aggressive sarcomas.

\section{Clinical case}

A 34-year-old man presented to our department with multiple asymptomatic skin lesions on his left leg. These lesions had appeared progressively over a 6-month period. There was no relevant personal or family history.

A clinical observation revealed multiple brownish papules and plaques on the lateral and medial sides of the left leg (Fig. 1). Some of these lesions had a hyperkeratotic surface and were tender and infiltrative on palpation. Because of the multifocality and the progressive appearance of newer lesions, a skin biopsy was performed on the same day.

Histologically, there was a moderately to highly cellular neoplasm, located predominantly on the subcutaneous tissue and extending to the dermis (Fig. 2). It was composed of a fascicular proliferation of plump spindle cells and epithelioid cells with prominent nucleoli and brightly eosinophilic cytoplasm, mimicking rhabdomyoblasts (Figs. 3 and 4).

The cells were arranged in disorganized bundles with a mod- erate inflammatory infiltrate of mononuclear predominance and dense collagen bundles. The mitotic index was low, with only 2 per 10 high-power fields identified. There was no evidence of necrosis.

Immunohistochemical analysis was performed and revealed strong and diffuse reactivity with keratins AE1/AE3, ERG, and FLI1 in both the spindle and the epithelioid neoplastic cells. Markers of muscular differentiation (desmin, myogenin, MyoD1, and smoothmuscle actin) and melanocytic markers (S10o-protein, HMB-45, and melan-A) were negative. Keratins MNF116 and CAM5.2, epithelial membrane antigen (EMA), CD31, and CD34 were also negative, as was CAMTA1, a recently described antibody expressed in epithelioid hemangioendothelioma (Table 1 and Fig. 5).

The nuclei of neoplastic cells showed intact INI1 expression (Table 1). The combination of the clinical, morphological, and immunophenotypical aspects was consistent with a diagnosis of PHE. Routine blood tests and infectious serology demonstrated no abnormalities. A CT scan of the chest, abdomen, and pelvis was unremarkable.

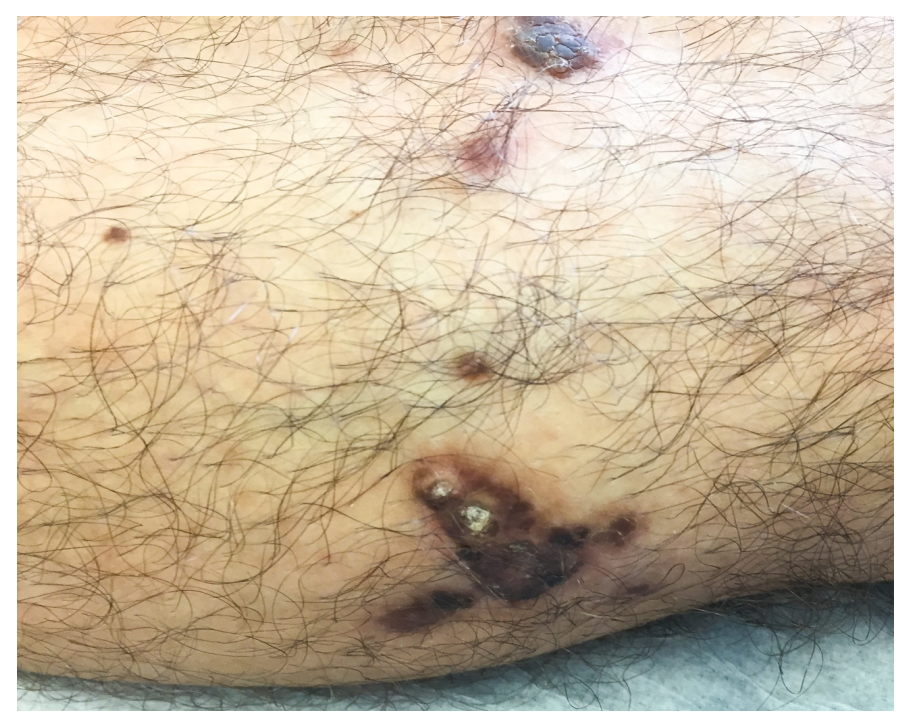

Figure 1 | Brownish papules and plaques on the left leg. 


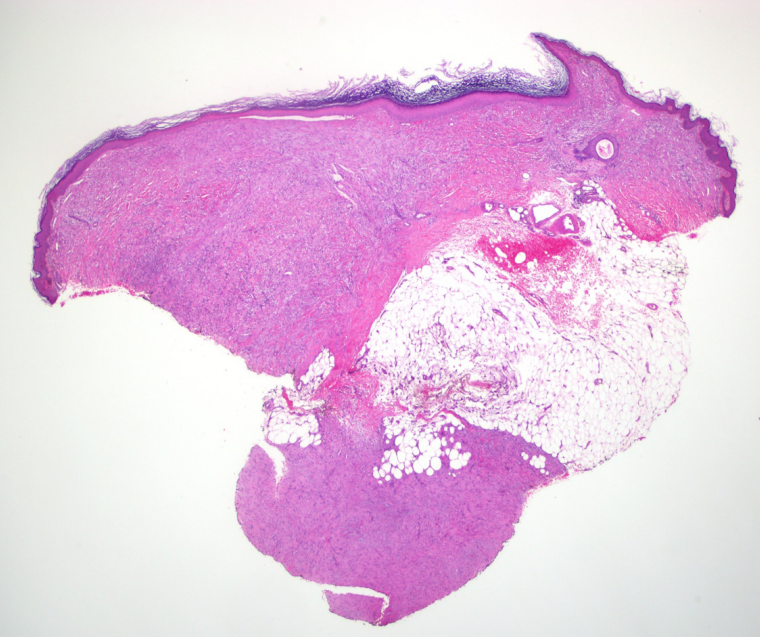

Figure $2 \mid \mathrm{H} \& \mathrm{E}$ (original magnification 20x). At scanning magnification, there is a dermal nodule with infiltration of the subcutaneous fat.
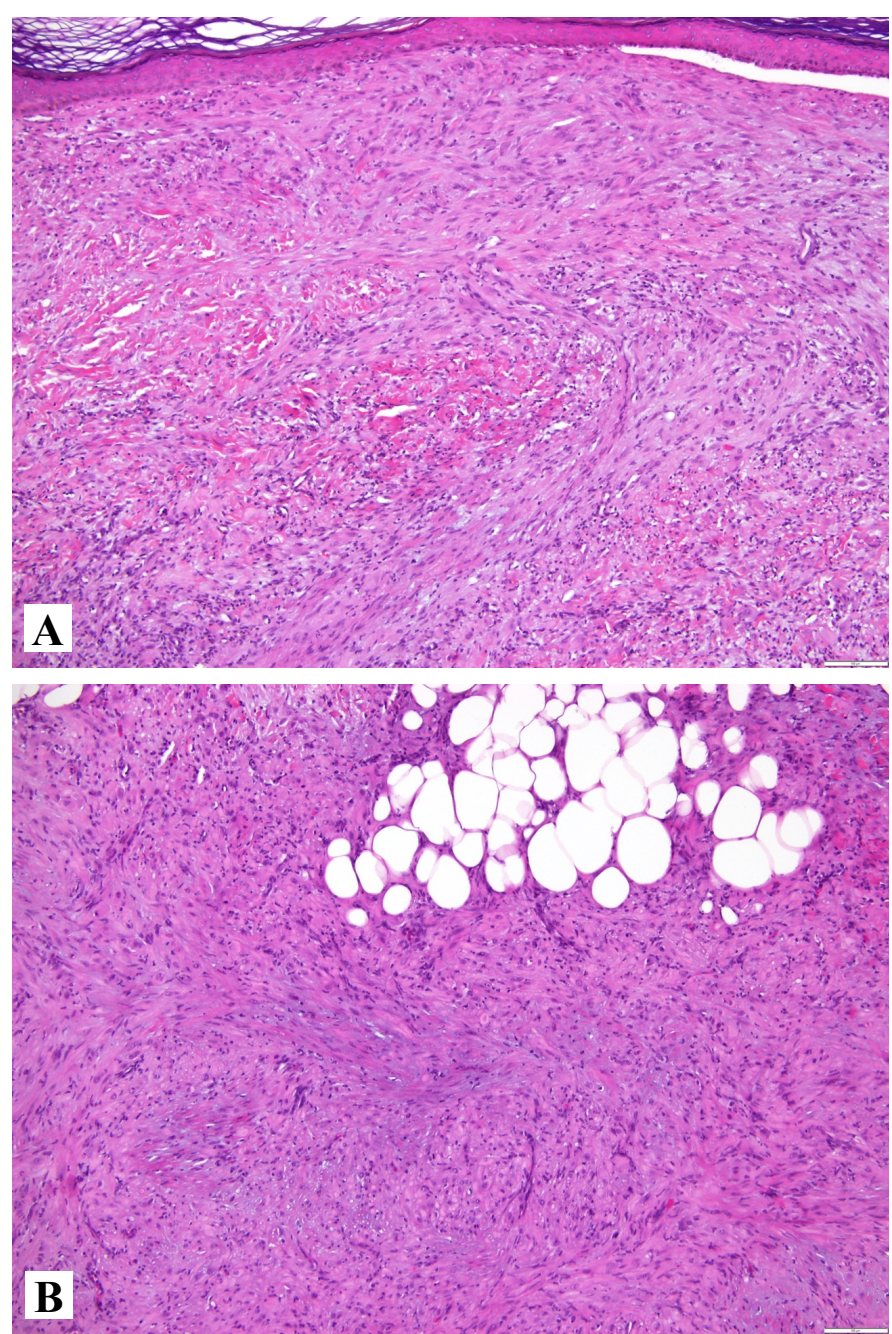

Figure $3 \mid$ H\&E (original magnification 100x). The nodule is composed of spindle cells with eosinophilic cytoplasm admixed with inflammatory cells (A) and epithelioid cells (B).

A CT scan of the lower limbs showed two well-defined nodular lesions in the skin-extending to the subcutaneous fat-in the anterior and medial aspects of the left leg, measuring $7 \mathrm{~mm}$ and 11 $\mathrm{mm}$, respectively. Several intracortical lytic lesions in the middle third of the left tibia, with dimensions varying from 1 to $5 \mathrm{~mm}$, were also found. A $3 \mathrm{~cm}$ nodule on the left soleus muscle was also suspected based on the scan.

After a multidisciplinary consultation with oncology, the pa- tient was sent to the local oncology center, where, due to the characteristic indolent behavior of the tumor, the patient undergoes strict and regular clinical and imagiological evaluations. Followup with an MRI occurs every 4 months. The surgical approach is held in reserve in the event the lesions grow markedly or symptoms such as pain appear between two follow-up consultations.

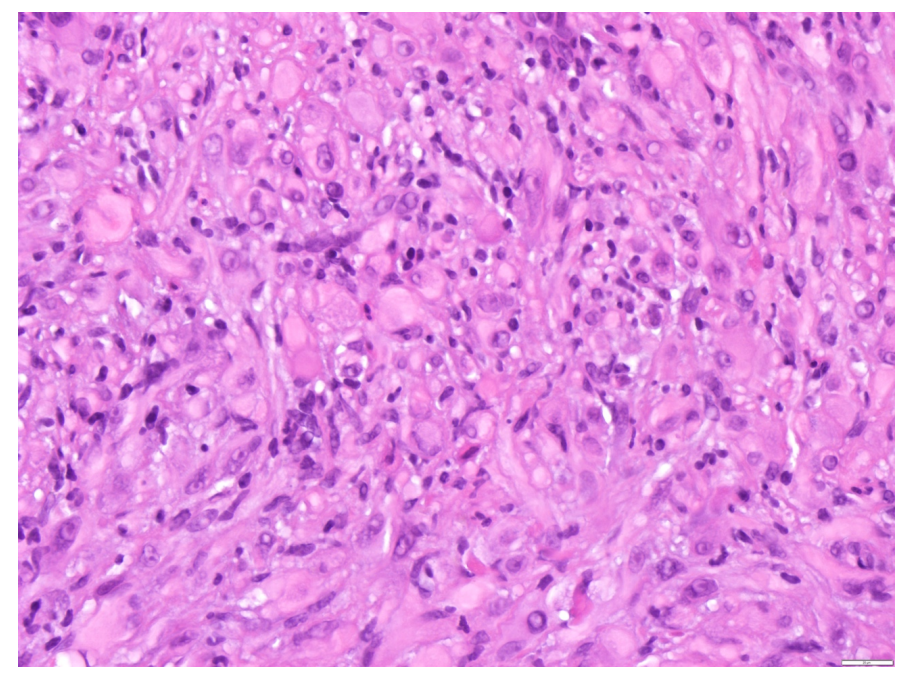

Figure 4 | H\&E (original magnification 400x). Note the epithelioid cells with glassy eosinophilic cytoplasm and prominent nucleoli, somewhat mimicking rhabdomyoblasts.

\begin{tabular}{lc} 
Table 1 | Immunophenotype of neoplastic cells in the case presented. \\
\hline Parameter & Value \\
\hline Pan-cytokeratin AE1/AE3 & + \\
Pan-cytokeratin MNF116 & - \\
Epithelial membrane antigen (EMA) & - \\
CD31 & - \\
CD34 & + \\
FLI1 & + \\
ERG & Focal \\
SMA & - \\
Desmin & - \\
MyoD1 & - \\
S100-protein & - \\
HMB-45 & - \\
Melan-A & - \\
MiTF & - \\
INI1 & + \\
CAMTA1 & - \\
\hline
\end{tabular}

\section{Discussion}

PHE is a rare tumor with fewer than 100 cases described in the literature. This tumor was probably first described by Mirra et al. in 1992 under the proposed name of fibroma-like variant of epithelioid sarcoma (4). It was thought to be related to epithelioid sarcoma because of its multifocal presentation on a single limb, often with osseous involvement, and its myoid cytomorphology with keratin-positive spindle cells. Additional cases resulted in a better understanding of the nature of PHE, and it is now recognized that it is not related to epithelioid sarcoma but rather is an independent neoplasm with an endothelial nature. In 2011, Hornick et al. proposed the name pseudomyogenic endothelioma, which better suits this entity (3).

PHE typically occurs in young males and tends to arise on the lower limbs, although it can also be found on the upper limbs, trunk, or face $\left(3,5^{-7}\right)$.

It is a clinically nonspecific tumor usually presenting as papules or nodules grouped in an anatomical region $(2,3,8-10)$. A 

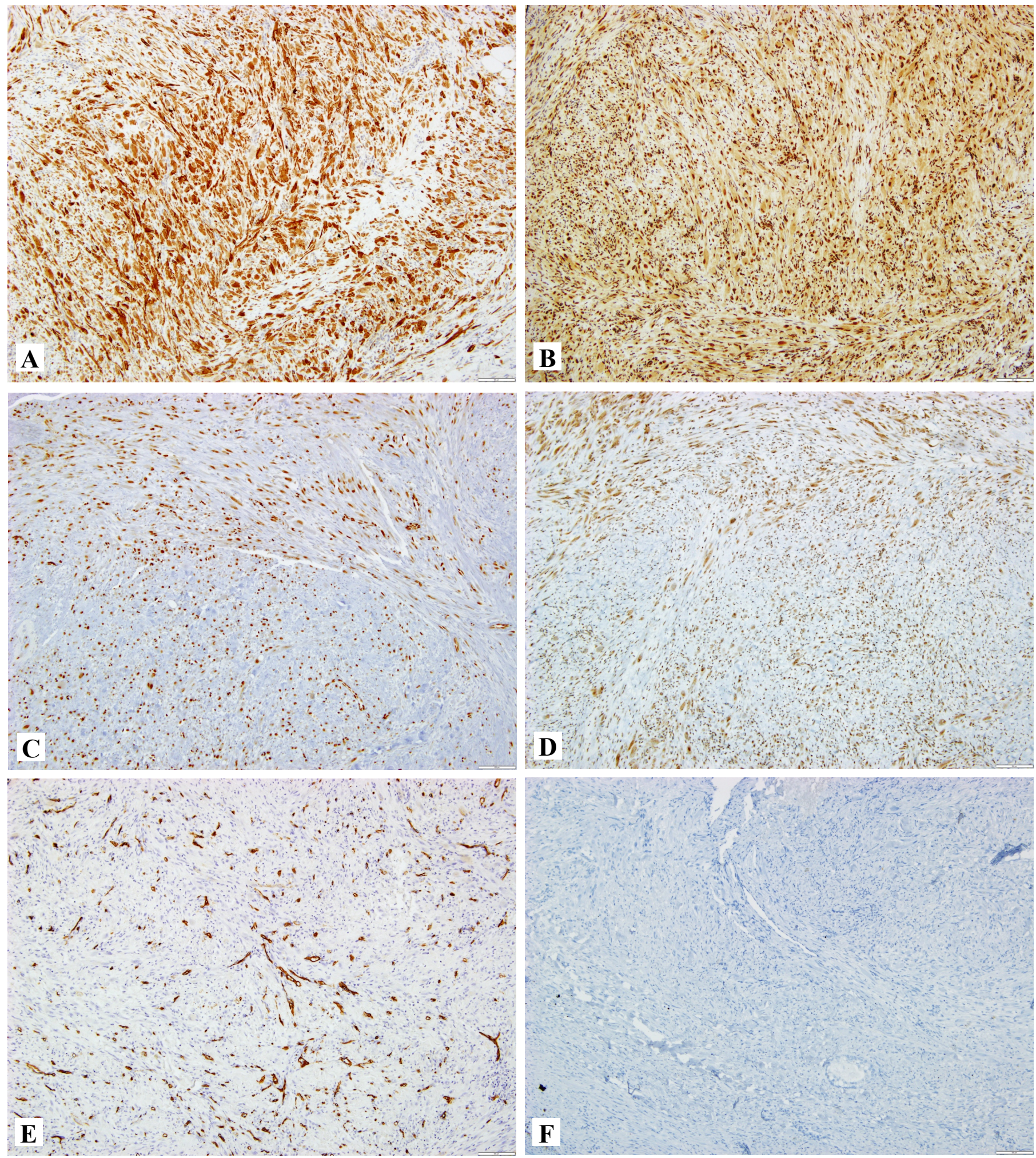

Figure 5 | Immunophenotype of neoplastic cells with immunoperoxidase technique (original magnification 100x). Both the epithelioid cells and spindle cells show diffuse immunoreactivity for keratin AE1/AE3 (A), FLI1 (B), and ERG (C). Unlike epithelioid sarcoma, there is intact expression of INI1 (D). There was no expression of CD34; note the expression in the endothelial cells as an internal control (E). Neoplastic cells were also negative for CAMTA1 (F).

multifocal presentation is found in two-thirds of patients at the time of diagnosis, and PHE often affects multiple tissue planes in the same anatomical region (11). Although the skin-primarily the dermis and the subcutis-is the most commonly affected tissue, about half of patients with PHE have muscular lesions and another $20 \%$ have osseous lesions (3). Approximately $50 \%$ of patients complain of painful nodules whereas the rest are asymptomatic (3). Due to the lack of specific clinical characteristics and the affliction of different body tissues, these patients are evaluated by multiple medical specialties, including dermatology, orthopedic surgery, and plastic surgery.

Histologically, PHE is an infiltrative neoplasm, involving the middle and deep reticular dermis and extending to the subcutaneous tissue. It is composed of fascicles of plump spindle cells and clusters of epithelioid cells that have the round, ample eosinophilic cytoplasm and prominent nucleoli characteristic of the so-called rhabdomyoblastic or pseudomyogenic morphology. Most tumors show mild to moderate cytological atypia, and mi- 
totic figures are usually low, usually less than 2 or 3 per 10 highpower fields (2-4).

Immunohistochemical analysis demonstrates the expression of keratins AE1/AE3, CD31, ERG, and FLI1 by both the spindle and the epithelioid neoplastic cells, with variable focal and weaker immunoreactivity for CAM5.2, smooth muscle actin, epithelial membrane antigen, and pan-cytokeratin MNF116, and usually no expression of $\mathrm{CD}_{34}$, desmin, and $\mathrm{S} 100$-protein.

In PHE the expression of INI1 (SNF5/SMARCB1) is intact, making it an important antibody for making the differential diagnosis with epithelioid sarcoma, which characteristically loses the reactivity with INI1. This differential diagnosis is particularly important because it has prognostic and therapeutic implications (2).

Although the histogenesis is still not clear, the expression of CD31, FLI1, and ERG-together with the clinical history and behavior-suggests that PHE might be a vascular neoplasm of intermediate biologic potential (3).

A specific SERPINE1-FOSB fusion derived from $\mathrm{t}(7 ; 19)(\mathrm{q} 22 ; \mathrm{q13})$ was identified and established PHE as a genetically distinct entity $(5,12,13)$. The strong nuclear expression of FOSB on immunohistochemistry in the tumor cells was demonstrated, enriching the panel of antibodies useful for the diagnosis of these tumors (5).

The main differential diagnoses of PHE include epithelioid sarcoma, epithelioid hemangioendothelioma, and epithelioid angiosarcoma.

Epithelioid sarcoma shares some clinical and morphologic features with PHE despite the different biologic behaviors. Epithelioid sarcoma metastasizes to the lymph nodes and lung in $40 \%$ to $50 \%$ of cases, whereas PHE shows a significant risk for locoregional recurrence but a low potential for distant metastasis (2). The immunohistochemical panel is quite distinct: PHE expresses CD31, ERG, FLI1, and FOSB, whereas epithelioid sarcoma is negative for these antibodies. Epithelioid sarcoma cells characteristically lose the expression of INI1 that remains intact in PHE neoplastic cells (12).

Epithelioid hemangioendothelioma is a low-grade malignant vascular neoplasm composed of anastomosing cords of epithelioid cells, with prominent cytoplasmic vacuolization, in a characteristic myxohyaline stroma (1). Epithelioid hemangioendothelioma usually expresses CD31, CD34, FLI1, and ERG, and shows reactivity for cytokeratins in 25 to $50 \%$ of tumors.

Epithelioid hemangioendothelioma has a characteristic trans-

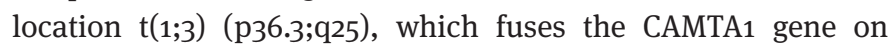
1p36.26 to the WWTR1 gene on 3q25.1 $(1,14)$. Additionally, the nuclear expression of CAMTA1 by immunohistochemistry is identi- fied in the majority of epithelioid hemangioendothelioma cases, whereas other epithelioid mesenchymal neoplasms, including pseudomyogenic hemangioendothelioma, are negative for CAMTA1 (13).

Epithelioid angiosarcoma is a high-grade malignant vascular tumor usually located in the deep soft tissue and composed of epithelioid endothelial cells with prominent atypia, a high nuclear grade, and frequent mitotic figures. Epithelioid angiosarcoma expresses vascular markers like CD31, CD34, ERG, and FLI1, and some tumors express cytokeratins, although this is usually a focal expression $(1,14)$.

The main treatment for PHE remains surgical excision of the lesions, even when there are multiple lesions, and sometimes amputation is needed to control the disease $(2,3,6,7,11,15)$. Related to the characteristic SERPINE1-FOSB fusion of PHE, IJzendoorn et al. recently reported the durable, complete remission of a patient with advanced unresectable PHE treated with telatinib (16). The authors concluded that telatinib (or other VEGFR1-4/PDGFRA inhibitors) could be a highly specific treatment option for patients with multifocal unresectable PHE (16).

Other less commonly used therapeutic options include radiotherapy-mostly postsurgical-and chemotherapy with various cytotoxic regimens and resulting in heterogenic responses $(2,3,11)$.

According to the latest WHO classification of soft tissues and bone tumors, PHE is a vascular tumor of intermediate malignancy that rarely metastasizes (1). Indeed, in the largest series of PHE described in the literature, only 1 out of 50 patients reported regional lymph node metastasis and only 1 out of 50 patients developed distant metastases and eventually died of the disease (3). The same study reported a recurrence rate of nearly $60 \%$, and the vast majority occurred within 1 year of the initial presentation.

In the light of this clinical behavior, long-term clinical and radiologic follow-up is recommended to determine the presence of new nodules or local recurrence $(3,6)$.

\section{Conclusion}

In conclusion, this case of PHE highlights its typical multifocal presentation in a young man, the lack of characteristic clinical findings, and the importance of histological and immunophenotypical study. Multiple medical specialists should be aware of the existence of this recently described entity. The authors present this case because of its rarity and to draw attention to the existence of this recently described and still poorly characterized tumor that often manifests with skin lesions.

\section{References}

1. Jo VY, Fletcher CD. WHO classification of soft tissue tumours: an update based on the 2013 (4th) edition. Pathology. 2014;46:95-104.

2. Requena L, Santonja C, Martinez-Amo JL, Saus C, Kutzner H. Cutaneous epithelioid sarcomalike (pseudomyogenic) hemangioendothelioma: a little-known low-grade cutaneous vascular neoplasm. JAMA Dermatol. 2013;149:459-65.

3. Hornick JL, Fletcher CD. Pseudomyogenic hemangioendothelioma: a distinctive, often multicentric tumor with indolent behavior. Am J Surg Pathol. 2011;35:190201.

4. Mirra JM, Kessler S, Bhuta S, Eckardt J. The fibroma-like variant of epithelioid sarcoma. A fibrohistiocytic/myoid cell lesion often confused with benign and malignant spindle cell tumors. Cancer. 1992;69:1382-95.

5. Sugita S, Hirano H, Kikuchi N, Kubo T, Asanuma H, Aoyama T, et al. Diagnostic utility of FOSB immunohistochemistry in pseudomyogenic hemangioendothelioma and its histological mimics. Diagn Pathol. 2016;11:75.
6. Amary MF, O'Donnell P, Berisha F, Tirabosco R, Briggs T, Pollock R, et al. Pseudomyogenic (epithelioid sarcoma-like) hemangioendothelioma: characterization of five cases. Skeletal Radiol. 2013;42:947-57.

7. Ye C, Yu X, Zeng J, Liu H, Dai M. Pseudomyogenic hemangioendothelioma secondary to fibrous dysplasia of the left lower extremity in a 14-year-old female: a case report. World J Surg Oncol. 2016;14:198.

8. Watabe A, Okuyama R, Hashimoto A, Hosaka M, Hatori M, Kariya Y, et al. Epithelioid sarcoma-like haemangioendothelioma: a case report. Acta Derm Venereol. 2009;89:208-9.

9. Tokyol C, Uzum N, Kuru I, Uluoglu O. Epithelioid sarcoma-like hemangioendothelioma: a case report. Tumori. 2005;91:436-9.

10. Billings SD, Folpe AL, Weiss SW. Epithelioid sarcoma-like hemangioendothelioma. Am J Surg Pathol. 2003;27:48-57. 
11. Joseph J, Wang WL, Patnana M, Ramesh N, Benjamin R, Patel S, et al. Cytotoxic and targeted therapy for treatment of pseudomyogenic hemangioendothelioma. Clin Sarcoma Res. 2015;5:22.

12. Hornick JL, Dal Cin P, Fletcher CD. Loss of INl1 expression is characteristic of both conventional and proximal-type epithelioid sarcoma. Am J Surg Pathol. 2009;33:542-50.

13. Doyle LA, Fletcher CD, Hornick JL. Nuclear expression of CAMTA1 distinguishes epithelioid hemangioendothelioma from histologic mimics. Am J Surg Pathol. 2016;40:94-102.
14. Hornick JL, Mangham DC. Annual review issue: soft tissue tumour pathology. Histopathology. 2014;64:1.

15. Karakasli A, Karaaslan A, Erduran M, Capkin S, Tuna EB, Havitcioglu H. Pseudomyogenic (epithelioid sarcoma-like) hemangioendothelioma with bone invasion. J Orthop. 2014;11:197-9.

16. van IJzendoorn DGP, Sleijfer S, Gelderblom H, Eskens FALM, van Leenders GJLH, Szuhai K, et al. Telatinib is an effective targeted therapy for pseudomyogenic hemangioendothelioma. Clin Cancer Res. 2018;24:2678-87. 